\title{
ANALISIS COMPARATIVO DE COSTO / RENDIMIENTO EN LA CONSTRUCCIÓN DE PLACA ENTREPISO MEDIANTE DOS METODOS CONSTRUCTIVOS DIFERENTES.
}

\author{
COMPARATIVE ANALYSIS OF \\ COST/PERFORMANCE IN THE \\ CONSTRUCTION OF MEZZANINE SLABS \\ USING TWO DIFFERENT CONSTRUCTION \\ METHODS.
}

\section{Javier Alfonso Cárdenas Gutiérrez ${ }^{1}$ \\ José Leonardo Jácome Carrascal ${ }^{2}$ \\ Mawency Vergel Ortega ${ }^{3}$}

Universidad Francisco de Paula Santander

\section{RESUMEN}

$1 \quad$ Magíster en Administración de Empresas con Especialidad en Dirección de Proyectos. Universidad Francisco de Paula Santander Cúcuta - Colombia, Orcid: 00000002-9894-0177 grupo de investigación en transporte $y$ obras civiles-GITOC Email: javieralfonsocg@ufps.edu.co 2 Magister en Ingeniería civil. Maestrante en educación matemática. Universidad Francisco de Paula Santander Cúcuta - Colombia, Orcid: https://orcid.org/ mawency@ufps.edu.co 0000-0002-9894-0177 jose.jacome@ufps.edu.co Grupo de investigación en estadística Graunt

3 Doctora en Educación. Postdoctora en Imaginarios y representaciones sociales. Docente y Directora del Departamento de Matemáticas y Estadística. Filiación: Universidad Francisco de Paula Santander. Correo electrónico: mawency@ufps.edu.co.

Orcid: https://orcid.org/0000-0001-8285-2968 Grupo de investigación en educación matemática Euler y grupo Graunt
En los últimos años los gobiernos han invertido en la realización en serie de viviendas con aras de satisfacer la demanda actual de personas sin hogar, en el caso de Colombia, victimas de desplazamiento por el conflicto armado, personas de escasos recursos, entre otras.

Por esta razón, los diferentes mandatarios han ido cumpliendo con la constitución nacional y los derechos fundamentales de entregar viviendas mínimamente dignas para la población en situación vulnerable.

De tal forma, las metodologías que se utilizan para la realización en serie de estas viviendas subsidiadas, deben medirse con el fin de 
encaminarse hacia una mejora continua, estableciendo procedimientos óptimos que permitan reducir los costos y los tiempos de entrega de este tipo de proyectos.

\section{PALABRAS CLAVE}

Vivienda de interés social, análisis de rendimientos, muros de carga, placas de entre piso, sistema industrializado.

\section{SUMMARY}

In recent years, governments have invested in the construction of housing in order to satisfy the current demand of homeless people, in the case of Colombia, victims of displacement due to the armed conflict, people of scarce resources, among others.

For this reason, the different leaders have been complying with the national constitution and the fundamental rights to provide minimally decent housing for the population in vulnerable situations.

Thus, the methodologies used for the serial implementation of these subsidized housing projects should be measured in order to move towards continuous improvement, establishing optimal procedures to reduce costs and delivery times of this type of projects.

\section{KEY WORDS}

Social interest housing, performance analysis, load-bearing walls, inter-floor slabs, industrialized system.

\section{INTRODUCCIÓN}

Con el comienzo de la consolidación urbana en Latinoamérica a mediados del siglo XX, los países han tenido que enfrentar los fenómenos de inequidad con respecto a la distribución de la riqueza. Estableciendo un equilibrio entre lo económico, lo social y lo cultural. Puesto que cada vez es más el crecimiento de la población urbana debido a la migración de los habitantes en zonas rurales hacia las ciudades, gracias al desarrollo de una cultura consumista e individualista. (Orozco Herrera \& López Toro, 2020)

Por lo tanto, desde hace muchos años el gobierno colombiano a buscado alternativas con aras de tratar estos casos inminentes de familias sin vivienda digna dentro de su territorio. Realizando esta labor con el fin de establecer una mejor calidad de vida a los ciudadanos con recursos más desfavorables, aquellas familias desplazadas por el conflicto armado o las personas que han sufrido calamidades domésticas, quedando sin el resguardo de una vivienda. (Puerto Castro, 2015)

El crecimiento poblacional en Colombia, durante los últimos años ha demarcado un déficit de personas sin hogar considerable, por lo cual, se ha visto en la necesidad de construir viviendas en serie, para así poder satisfacer de manera paulatina la alta demanda de familias sin un techo para vivir.(Mosquera Ayala \& Noreña Trejos, 2021)

Por esta razón, se debe incentivar la optimización en este tipo de producción en masa, así como en cada uno de los sistemas que las conforman (Villanueva Joaquin \& Bustos Tirado, 2020). Hoy en día se cuentan con distintos métodos constructivo los cuales poseen una serie de mecanismos que permiten realizar viviendas en serie bajo las normas establecidas, dos de estos mecanismos que utilizan las empresas son el sistema constructivo tradicional y el industrial con elementos prefabricados. (Tovar, 2021)

Estas metodologías difieren en rendimiento, siendo este el gasto de materiales, mano de obra y tiempo (Cárdenas-Gutiérrez et al., 2020) metodologías que permiten estimar el costo total y posteriormente determinar una programación de obra lo más precisa posible (Matíz Beltrán \& Rueda Gómez, 2020). Dichos métodos, pueden 
Ilegarse a calcular mediante actividades similares que permitan determinar una relación directa y a su vez poder comparar la mejor relación costo/ rendimiento entre procedimientos.

Es importante que dentro de la industria de la construcción se evalúen todas las alternativas posibles, no solo con el fin de conocer sus costos, sino de poder tener una capacidad analítica y desarrollar la destreza para tomar las mejores decisiones y que estas lleven a la mejor solución según el contexto (Riaño Anaya, 2021)

De tal forma, que no solo en los proyectos de vivienda, sino en todo tipo de obra civil, se debe realizar un seguimiento riguroso, desde la ejecución de la actividad hasta su traducción numérica real, siendo este el indicativo claro de la eficiencia con la que se está llevando a cabo cualquier labor (Aguilar Aguirre, 2021).

El objetivo de este trabajo es poder establecer una relación entre el costo y el rendimiento del proceso constructivo en las placas de entre piso mediante dos metodologías diferentes en dos proyectos de vivienda de interés social en la ciudad de Cúcuta - Norte de Santander, Colombia. Con los resultados obtenidos se podrá establecer cuál es el método constructivo más factible con base a los requisitos del proyecto y el contexto de la ciudad de Cúcuta.

\section{METODOLOGÍA}

La metodología empleada en el siguiente trabajo abarca:

1. Conceptualizaciones.

2. Calculo de rendimientos en formaletas para viviendas en serie mediante el método constructivo tradicional.

3. Calculo de rendimientos en formaletas para viviendas en serie mediante el método constructivo industrializado.
4. Comparativa entre el costo y el rendimiento entre los dos métodos constructivos utilizados.

\section{DESARROLLO}

\section{Conceptualizaciones}

Sistema tradicional: Son los métodos utilizados de manera convencional, que tienen un grado bajo de industrialización, teniendo como factor fundamental el conocimiento empírico, la mano de obra y la mampostería simple (Housing, 2015)

Sistema industrializado : Son los métodos utilizados para la producción en serie de elementos estructurales, tales como muros y placas, aportando un mayor rendimiento en los tiempos de ejecución, basados en una formaleta modulada para la construcción en serie (Romero \& Vaquiro, 2016).

Vivienda de interés social : Es aquel tipo de vivienda que reúne los diferentes elementos que aseguran su habitabilidad, sus estándares de calidad con respecto al diseño urbanístico, arquitectónico y de construcción.(Amico Benvenuto \& Amico León, 2021)

\section{Cálculo de rendimientos en formaletas para viviendas en serie mediante el método constructivo tradicional}

Los datos para realizar el cálculo del rendimiento se obtuvieron mediante un seguimiento a las tres actividades principales a evaluar, las cuales son: encofrado de formaleta, vaciado de concreto y desencofrado de formaleta.

Como datos primarios se consideraron el tipo y la cantidad de formaleta utilizada, el tiempo usado en cada una de las actividades, la cuadrilla que intervino en el proceso y el área o volumen sobre el cual se realizará cada una de estas actividades. Como datos secundarios se consideraron, el tipo, cantidad y especificaciones 
del concreto, la herramienta mejor y el método utilizado para su fundida. Los datos obtenidos corresponden a 5 casas construidas en el primer proyecto de vivienda de interés social mediante la metodología tradicional.

Mediante el uso de un formato diseñado para la obtención de datos con respecto al método constructivo tradicional se logró hacer seguimiento a cada una de las sub actividades que afecta directamente el tiempo de utilización de esta formaleta, teniendo en cuenta, tiempo de armado, acero, vaciado, fraguado y desencofrado, especificando la duración de cada una, con el fin de obtener un promedio del tiempo total de uso de esta formaleta. En la tabla 1 se logra evidenciar los valores promedios de este seguimiento para las 5 casas.

TRADICIONAL EN MAMPOSTERIA

\begin{tabular}{c|c|c|}
\hline METODO CONSTRUCTIVO & UNIDAD & PROMEDIO \\
\hline Área de Placa & M2 & 83,313855 \\
Volumen de Placa & M3 & 16,68771 \\
Armado de Formaleta & H & 12,546 \\
Armado de aceros & H & 12,24 \\
Aligerante Bloque \#5 & H & 3,06 \\
Tiempo total de Vaciado & H & 0,25245 \\
Codal & H & 0,173 \\
Fraguado & H & 91,8 \\
Desencofrado & H & 5,5 \\
Curado & H & 2,1 \\
Cuadrilla & Oficial + & 7 \\
Cuadrilla Menor & Ayudantes & \\
Cantidad de concreto & Oficial + & 2 \\
Cantidad de formaleta & Myudante & 7,5 \\
Cantidad de Parales & UND & 74 \\
Cantidad de Cerchas & UND (3m) & 104 \\
\hline
\end{tabular}

Tabla 1. Subactividades Método Tradicional

Adicionalmente, en la tabla 2 se muestran los diferentes aceros utilizados en las vigas y viguetas para la construcción de la casa entrepiso.

\begin{tabular}{cccc}
\hline $\begin{array}{c}\text { TIPO DE } \\
\text { ACERO }\end{array}$ & $\begin{array}{c}\text { CANTIDAD } \\
\text { (KG) }\end{array}$ & $\begin{array}{c}\text { TIEMPO } \\
\text { (HORAS) }\end{array}$ & $\begin{array}{c}\text { CUADRILLA } \\
\text { ( \# DE } \\
\text { HOMBRES) }\end{array}$ \\
$\begin{array}{c}\text { Flejes @ } \\
5 / 16\end{array}$ & 337,10 & 5 & 2 \\
Varilla \#3 & 516,57 & 6 & 2 \\
Varilla \#4 & 19,16 & 3 & 2 \\
Varilla \#5 & 177,73 & 3 & 2 \\
\hline
\end{tabular}

Tabla 2. Aceros utilizados en método tradicional 
Teniendo en cuenta que rendimiento es la cantidad de medida realizada de una actividad con respecto a la composición de la cuadrilla, expresada en unidades de hora/hombre (Hh) (Rojas Torres, 2020)

Por lo tanto, tenemos la ecuación 1:

$$
\begin{gathered}
\text { Rendimiento }=\frac{\text { Cantidad de personas } x \text { Duracion de actividad }}{\text { Cantidad de obra ejecutada }} \\
\text { Rendimiento }=\frac{\text { Hora Hombre }}{\text { Unidad }}
\end{gathered}
$$

A continuación, se explica detalladamente un ejemplo de cómo se realiza este cálculo, y este procedimiento se replicará con cada actividad.

Rendimiento Formaleta $=\frac{7 \text { hombres } x 12,5 \mathrm{H}}{83,313855 \mathrm{~m}^{2}}=1,05025 \frac{\mathrm{Hh}}{\mathrm{m}^{2}}$

En la tabla 3 se puede notar el rendimiento de cada actividad.

\begin{tabular}{|c|c|c|}
\hline \multicolumn{3}{|c|}{ RENDIMIENTOS METODO TRADICIONAL } \\
\hline ACTIVIDAD & RENDIMIENTO & UNIDAD \\
\hline $\begin{array}{c}\text { Armado de } \\
\text { formaleta }\end{array}$ & 1,05025 & $\mathrm{Hh} / \mathrm{m}^{2}$ \\
\hline Armado de flejes & 0,029665 & $\mathrm{Hh} / \mathrm{kg}$ \\
\hline Armado de acero\#3 & 0,02323 & $\mathrm{Hh} / \mathrm{kg}$ \\
\hline Armado de acero\#4 & 0,313152 & $\mathrm{Hh} / \mathrm{kg}$ \\
\hline Armado de acero\#5 & 0,033759 & $\mathrm{Hh} / \mathrm{kg}$ \\
\hline Vaciado & 0,23562 & $\mathrm{Hh} / \mathrm{m}^{3}$ \\
\hline Codal & 0,004152971 & $\mathrm{Hh} / \mathrm{m}^{2}$ \\
\hline Desencofrado & 0,462108 & $\mathrm{Hh} / \mathrm{m}^{2}$ \\
\hline Curado & 0,05041178 & $\mathrm{Hh} / \mathrm{m}^{2}$ \\
\hline
\end{tabular}

Tabla 3. Rendimientos método tradicional

\section{CALCULO DE RENDIMIENTOS EN FORMALETAS PARA VIVIENDAS EN SERIE MEDIANTE EL MÉTODO CONSTRUCTIVO INDUSTRIALIZADO}

En el segundo proyecto se realizó el seguimiento de toda la etapa estructural de estas viviendas, a diferencia del proyecto anterior las formaletas utilizadas fueron de acero, y el tipo de placa fue maciza, así como el acero empleado fue por medio de mallas electrosoldadas.
La toma de datos se realizó de la misma forma que con el método anterior, recolectando datos de 5 viviendas también de las cuales posteriormente se determinó un promedio, con el fin de poder calcular los rendimientos.

Por lo tanto, en la tabla 4 se encuentran los promedios del seguimiento realizado a estas viviendas. 


\begin{tabular}{ccc}
\hline \multicolumn{3}{c}{ METODO INDUSTRIALIZADO } \\
\hline METODO CONSTRUCTIVO & UNIDAD & PROMEDIO \\
Área de Placa & M2 & 33,46 \\
Volumen de Placa & M3 & 4,2 \\
Armado de Formaleta & $\mathrm{H}$ & 3,6 \\
Armado malla electrosoldada & $\mathrm{H}$ & 2,7 \\
Kg de Malla empleados & $\mathrm{KG}$ & 122,5 \\
\hline Tiempo total de Vaciado & $\mathrm{H}$ & 0,1 \\
Codal & $\mathrm{H}$ & 0,22 \\
Fraguado & $\mathrm{H}$ & 14,2 \\
Desencofrado & $\mathrm{H}$ & 2,7 \\
Curado & $\mathrm{H}$ & 0,76 \\
Cuadrilla & Oficial + & 9 \\
Cuadrilla Menor & Ayudantes & \\
Especificaciones del concreto & Oficial + & 2 \\
Cantidad de concreto & Ayudante & $3000 \mathrm{psi}$ \\
Cantidad de formaleta & M3 & 7 \\
Cantidad de Parales & UND & 61 \\
Cantidad de Cerchas & UND (3m) & 143 \\
Cantidad de Cerchas & UND $(2 \mathrm{~m})$ & 21 \\
\hline
\end{tabular}

Tabla 4. Subactividades Método Industrializado

Utilizando la misma ecuación que en el cálculo de los rendimientos del proyecto anterior, tenemos los siguientes resultados en la tabla 5 :

\begin{tabular}{|c|c|c|}
\hline \multicolumn{3}{|c|}{ RENDIMIENTOS METODO INDUSTRIALIZADO } \\
\hline ACTIVIDAD & RENDIMIENTO & UNIDAD \\
\hline $\begin{array}{l}\text { Armado de } \\
\text { formaleta }\end{array}$ & 0,968320 & $\mathrm{Hh} / \mathrm{m} 2$ \\
\hline $\begin{array}{l}\text { Amaro de malla } \\
\text { electrosoldada }\end{array}$ & 0,04408163 & $\mathrm{Hh} / \mathrm{kg}$ \\
\hline Vaciado & 0,214285714 & $\mathrm{Hh} / \mathrm{m} 3$ \\
\hline Codal & 0,01315003 & $\mathrm{Hh} / \mathrm{m} 2$ \\
\hline Desencofrado & 0,7262402 & $\mathrm{Hh} / \mathrm{m} 2$ \\
\hline Curado & 0,04542737 & $\mathrm{Hh} / \mathrm{m} 2$ \\
\hline
\end{tabular}


Tabla 5. Rendimientos método industrializado

COMPARATIVA ENTRE EL COSTO Y EL RENDIMIENTO ENTRE LOS DOS MÉTODOS CONSTRUCTIVOS UTILIZADOS.

La relación del costo total del alquiler de la

hidráulicas y eléctricas, se funde la placa, fragua y se desencofra.

\begin{tabular}{ccc|ccc}
\hline \multicolumn{4}{c}{ ALQUILER EQUIPOS METODO TRADICIONAL } \\
\hline EQP. & UND & CANT. & V. DíA & $\begin{array}{c}\text { SUB } \\
\text { TOTAL }\end{array}$ \\
Tableros & Und & 74 & $\$ 125$ & $\$ 9.250$ \\
Paral & Und & 104 & $\$ 102,50$ & $\$ 10,660$ \\
Cercha & Und & 43 & $\$ 106,28$ & $\$ 4,570$ \\
\hline \multicolumn{4}{c}{ VALOR TOTAL ALQUILER X DÍA } & $\$ 24.480$ \\
\hline \multicolumn{7}{l}{ VALOR TOTAL ALQUILER X 20 DÍAS } & $\$ 489.600$ \\
\hline
\end{tabular}

Tabla 5. Costos método tradicional.

EQP:Equipos, V.Dia: Unidad día. CANT: Cantidad,

A continuación, se expone la relación del aproximadamente 4 días, el tiempo total en el que se arma la formaleta, se instala la malla electrosoldada, se instala la red hidráulica, se

realiza el vaciado de concreto y se desencofra en una vivienda.

\begin{tabular}{ccc|cl}
\hline \multicolumn{4}{c|}{$\begin{array}{c}\text { ALQUILER EQUIPOS METODO } \\
\text { INDUSTRIALIZADO }\end{array}$} \\
\hline EQP. & UND & CANT. & $\begin{array}{c}\text { V. } \\
\text { DÍA }\end{array}$ & SUB TOTAL \\
Formaleta & M2 & 205 & $\$ 602$ & $\$ 23.410$ \\
metálica & & & $\$ 126$ & $\$ 18.017,5$ \\
Paral & Und & 143 & $\$ 110$ & $\$ 2.310,00$ \\
Cercha & Und & 21 & $\$ 110$ \\
Cercha & Und & 21 & $\$ 110$ & $\$ 2.310,00$ \\
\hline \multicolumn{3}{l}{ VALOR TOTAL ALQUILER X DÍA } & $\$ 146.047,50$ \\
\hline \multicolumn{3}{l}{ VALOR TOTAL ALQUILER X 4 DÍAS } & $\$ 584.190,00$ \\
\hline \multicolumn{4}{l}{}
\end{tabular}

Tabla 6. Costos método industrializado

Posteriormente, al calcular los rendimientos de cada una de las sub actividades, en los proyectos, basados en el tiempo, otros datos y las observaciones, se determina que en este estudio específico los rendimientos obtenidos en Hh/unidad son directamente proporcionales al tiempo empleado en cada una de las subactividades, lo que lleva a la conclusión de que a mayor tiempo de rendimiento menor es la productividad y la eficiencia.

Estos valores calculados, se puede observar en 
la figura 1, donde se establece el valor lineal de los costos con respecto al tiempo, notando un valor muy similar, pero en tiempos de ejecución diferentes.

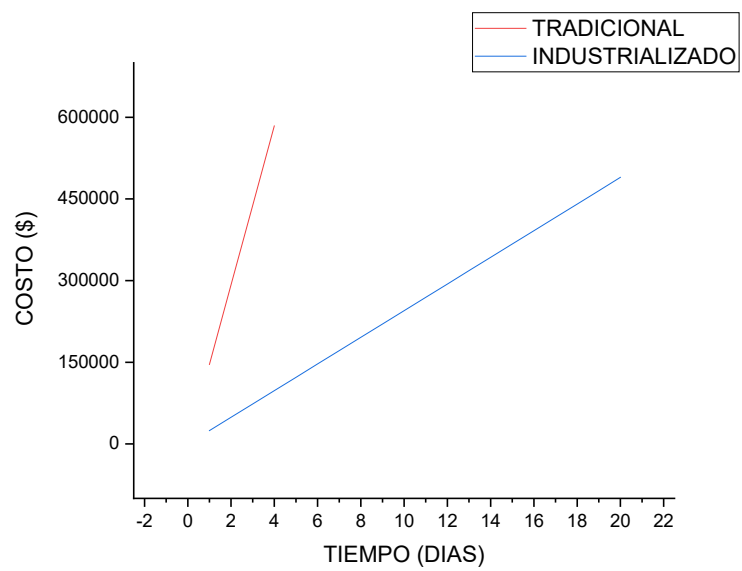

Figura 1. Tiempo vs costo de ambos métodos.

En la Figura 2, podemos observar mediante un gráfico de barras, los valores que se encuentran en la tabla 3 y 5 .

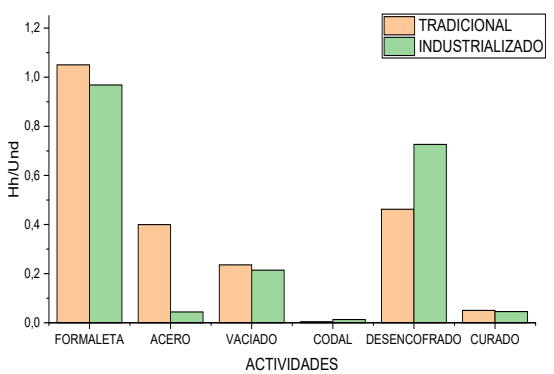

Figura 2. Comparación rendimientos ambos métodos.

\section{CONCLUSIONES}

Basados en el seguimiento realizado a los proyectos constructivos se demuestra que es más eficiente construir por el método industrializado siempre que el tiempo sea un factor limitante en el desarrollo del proyecto.

Sin embargo, con base a lo calculado, se logró observar que el armado de la formaleta es un $8,4 \%$ más eficiente en el método industrializado, adicionalmente la realización y colocación de las mallas electrosoldadas es 9 veces más rápida que el figurado de acero de manera tradicional, con respecto al vaciado del concreto en el método industrializado es cerca de un $10 \%$ más eficiente, el rendimiento con los codales es tres veces más eficiente en el método tradicional, el desencofrado un $57 \%$ más eficiente en el método tradicional y el curado representa un $10 \%$ más de eficiencia en el método industrializado.

Por lo tanto, no es posible encontrar un punto claro de comparación entre el método tradicional y el método industrializado debido a la gran diferencia que existe entre estos en cuanto a la utilización de las formaletas y la metodología desarrollada en las subactividades que afectan el tiempo de uso de este equipo. Se podría decir que la diferencia más notable es con base al tiempo de ejecución total de las actividades, puesto que en la relación Tiempo vs Costo, se aprecia que el método tradicional tarda 5 veces más que el método industrializado.

De tal forma, se concluye que cada proyecto es independiente y presenta características individuales, por lo cual cada método debe ser estudiado de forma independiente teniendo en cuenta cada una de las fases de desarrollo para de esta forma poder mejorar sus rendimientos $y$ por ende su productividad buscando confort de todas las partes involucradas.

Finalmente, la relación rendimiento - costo, termina dependiendo del tiempo disponible dentro de la programación establecida en el cronograma, puesto que ambos poseen un costo similar y solo en caso de necesitar realizar esta actividad de manera urgente, se utilizaría el método industrializado. 


\section{REFERENCIAS BIBLIOGRÁFICAS}

Aguilar Aguirre, J. P. (2021). Seguimiento y análisis de los factores que afectan el rendimiento en la excavación del túnel de conducción del proyecto hidroeléctrico TZ 2.

Amico Benvenuto, A., \& Amico León, A. A. (2021). Determinación de los costos del concreto premezclado para las viviendas de interés social de la ciudad de Piura, 2018.

Cárdenas-Gutiérrez, J., Molina-Salazar, L., \& Medrano Lindarte, C. T. (2020). Costos y Presupuetos en la Industria de la Construcción. Eco Ediciones.

Housing, O. (2015). Construcción Industrializada versus Construcción Tradicional, ventajas e inconvenientes. https://www.oboxhousing.

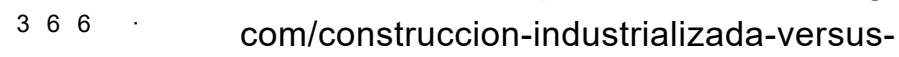
construccion-tradicional/

Matíz Beltrán, N. K., \& Rueda Gómez, L. N. (2020). Manual para estimar el tiempo requerido en la construcción de vivienda. Universidad Cooperativa de Colombia, Facultad de Ingenierías, Ingeniería ....

Mosquera Ayala, L., \& Noreña Trejos, M. A. (2021). Estudio de viabilidad ambiental y financiero sobre las técnicas de la construcción sostenible que pueden adoptarse en la construcción tradicional de viviendas en Colombia.

Orozco Herrera, D. C., \& López Toro, A. (2020). Déficit habitacional y pobreza: un abordaje desde las políticas públicas de vivienda en Colombia. Caso Cien mil viviendas gratis para los colombianos y Tarso mi techo solidario (2012-2019)(dossier).

Puerto Castro, O. M. (2015). Informe de pasantía en el proyecto de vivienda centro residencial Avatar. Universidad Santo Tomas.

Riaño Anaya, L. X. (2021). Manejo financiero de una empresa de servicios dedicada al alquiler de equipos para construcción ubicada en la ciudad de Duitama-Boyacá.

Rojas Torres, L. K. (2020). Análisis de rendimientos de equipos en el proceso de perforaciones de anclajes para taludes según el tipo de suelo e imprevistos.

Romero, C. Y., \& Vaquiro, E. M. (2016). MANUAL DE CONSTRUCCION PARA VIVIENDA DE INTERES SOCIAL CON SISTEMAS INDUSTRIALIZADOS TIPO MANOPORTABLE Y TÚNEL.

Tovar, A. (2021). Construcción industrializada, el cambio necesario para los nuevos desafíos. Cemento Hormigón, 1003, 3.

Villanueva Joaquin, L. E., \& Bustos Tirado, J. O. (2020). Optimización de los procesos productivos utilizando Value Stream Mapping (VSM) en los procesos constructivos de placa de ascensor, placa de escalera y losa maciza "sector 4": Caso: Proyecto "Condominio Bolivia $n^{\circ} 848$ " Breña-Lima. 